\title{
Behavior and dynamics of gecko's locomotion: The effects of moving directions on a vertical surface
}

\author{
WANG ZhouYi ${ }^{1,2}$, WANG JinTong ${ }^{1,2}$, JI AiHong $^{1}$, ZHANG YueYun $^{3}$ \& DAI ZhenDong ${ }^{1 *}$ \\ ${ }^{1}$ Institute of Bio-inspired Structure and Surface Engineering, Nanjing University of Aeronautics and Astronautics, Nanjing 210016, China; \\ ${ }^{2}$ College of Mechanical and Electrical Engineering, Nanjing University of Aeronautics and Astronautics, Nanjing 210016, China; \\ ${ }^{3}$ Guangxi Botanical Garden of Medicinal Plants, Nanning 530023, China
}

Received January 4, 2010; accepted May 5, 2010

\begin{abstract}
The study of the movement behavior of geckos on a vertical surface, including the measurement and recording of the reaction forces as they move in different directions, plays an important role in understanding the mechanics of the animals' locomotion. This study provides inspiration for the design of a control system for a bionics robot. The three-dimensional reaction forces of vertical surface-climbing geckos (Gekko gecko) were measured using a three-dimensional force-sensors-array. The behavior of gecko as it moved on a vertical surface was recorded with a high speed camera at $215 \mathrm{fps}$ and the function of each foot of a gecko are discussed in this paper. The results showed that the gecko increased its velocity of movement mainly by increasing the stride frequency in the upward, downward and leftward direction and that the speed had no significant relationship to the attachment and detachment times. The feet above the center-of-mass play a key role in supporting the body, driving locomotion and balancing overturning etc. The movement behavior and foot function of geckos change correspondingly for different conditions, which results in safe and effective free vertical locomotion. This research will be helpful in designing gecko-like robots including the selection of gait planning and its control.
\end{abstract}

gecko, three-dimensional reaction force, movement behavior, vertical surface, sensors-array

Citation: Wang Z Y, Wang J T, Ji A H, et al. Behavior and dynamics of gecko locomotion: The effects of moving directions on a vertical surface. Chinese Sci Bull, 2011, 56: 573-583, doi: 10.1007/s11434-010-4082-7

In unstructured environments, modern robots are quite inferior to animals with regard to stability, agility, robustness, environmental adaptability, and energy efficiency [1]. Studying the reaction forces between the foot of an animal and the substrate on which it is moving, as well as the locomotion behaviors and its relationship with the reaction force, will help us to understand the mechanical roles of animal locomotion. This in turn will provide inspiration for structure design, gait planning and control system development for a bio-mimicking-robot. The results show that scansorial animals often require different locomotive apparatus when compared with ground-dwelling animals [2-5]. Level runners usually have relatively long limbs to elevate their body

*Corresponding author (email: zddai@nuaa.edu.cn) from the ground to reduce friction and move more sagittally to increase stride length. However, climbers benefit from shorter limbs and use a sprawling gait to keep their centerof-mass as close to the substrate as possible [5-12]. When animals move over a steep or vertical surface, they must generate sufficient propulsive forces to overcome inertia, surface resistances, air and gravity [6,10]. On inclines, the fore-feet must generate an adhesive force to avoid overturning backwards while the hind-feet provide the propulsion needed for locomotion [10]. Geckos have been widely researched by scientists because of their efficient and versatile adhesive abilities. Damme et al. focused on the behavior and dynamics of geckos moving on floors, walls and ceilings [11,13-18]. Losos et al. have carried out significant research on the morphology $[3,6,9]$, structure and micro-structure of 
gecko feet [19-21], mechanisms of adhesion [19,22-24], and adhesive strength [20,25-28]. On bionic applications, the artificial seta array was developed by micro-mold injection [29,30], electron-beam etching [31], and carbon nanotubes [32].

The motion properties of a gecko on a vertical surface have been well researched [5,13-15,33], but the relationship between the reaction forces and relative direction of movement has not been studied. Here we, for the first time in literature, measured the three dimensional reaction forces and observed the locomotion behavior of freely moving geckos on a vertical surface in three directions (upward, downward and leftward). We believe the results will provide biological knowledge for an engineer to develop an advanced gecko-mimicking robot.

\section{Materials and methods}

(i) Animals. Five geckos from Guangxi Zhuang Autonomous Region, China were used in this experiment (body weight: $64.5 \pm 2.4 \mathrm{~g}$, body length: $136.6 \pm 12.4 \mathrm{~mm}$, mean \pm s.d.). The geckos were housed in two cages which were connected by an aisle to train the gecko to move through the aisle of the force-sensors-array to feed on mealworms, crickets, vitamins and water. They were kept under a natural light cycle, the temperature at $25 \pm 2^{\circ} \mathrm{C}$, humidity at $60 \%$
$-70 \%$. To make the description easy, we defined each foot as LF, LH, RF and RH (Figure 1(a)).

(ii) Experimental setup. The facility was made up of a sensor array at the bottom of an aisle to measure the reaction force between the foot and the substrate (load-carrier of the sensor), which was covered with transparent Plexiglas to limit the motion of the gecko directly onto the force-sensor-array. Two mirrors at $45^{\circ}$ to the force-sensorarray (FSA) plane enabled the recording of the three-dimensional locomotion behavior of the gecko's motion. The FSA platform was made up of $16(2 \times 8)$ sensors and each sensor could measure the three-dimensional interaction forces. The end of the aisle had a dark plastic box to lure the gecko to move into it (Figure 1(a)). The FSA platform rotated about an axis to change the direction of the aisle to simulate gecko movement on the vertical surface in upward, downward and leftward directions (Figure $1(\mathrm{c}))$.

(iii) Force measurements and acquisitions. The threedimensional reaction force (Figure 1(b)), namely, lateral, fore-aft and normal forces, when the geckos moved freely on the vertical surface, were measured and collected by the experimental facility described above. Three-dimensional sensors with micro-Newton resolution measured the reaction forces [34]. The strains were regulated by a signal processing system (SCXI-1520 NI, USA), and digitized by (a)

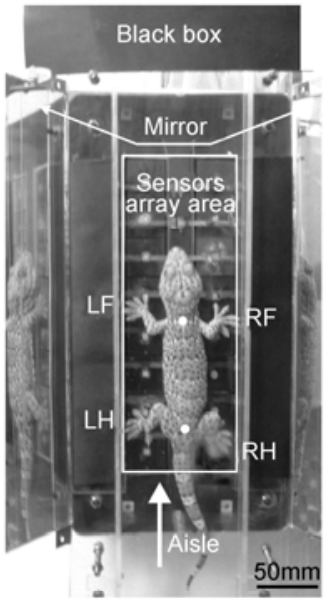

(b)

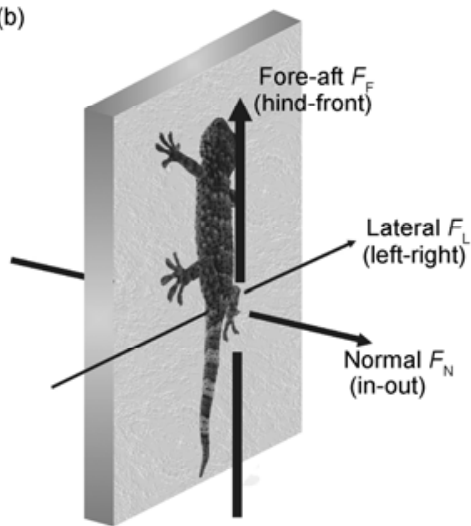

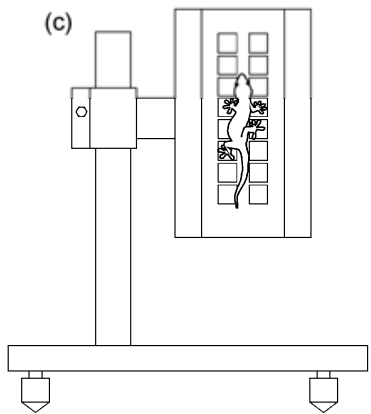

(1)

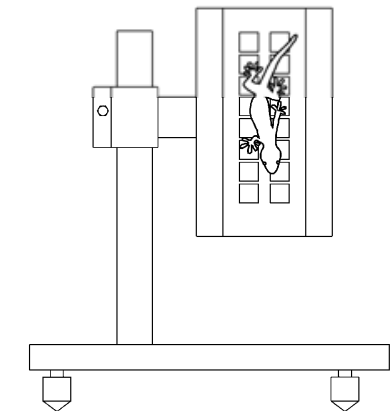

(2)

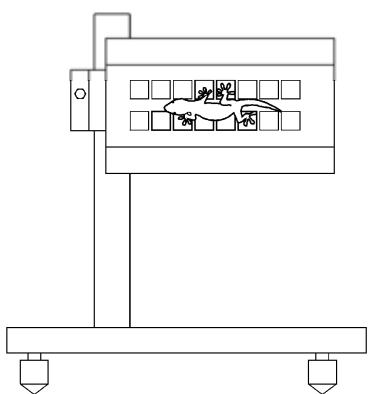

(3)

Figure 1 (a) Force platform used to measure dynamics of G. Gecko moving on a vertical surface; (b) directional definition of reaction forces in this research; (c) diagrams of gecko moving in different directions on a vertical surface: (1) upward; (2) downward; (3) leftward. 
an acquisition card (PCI-6052E, NI, USA). The collected data were filtered at a cut-off frequency of $100 \mathrm{~Hz}$. The software was developed in our laboratory on a LabVIEW platform.

(iv) Kinematics observation. The locomotion behavior of the geckos was recorded using a high speed camera at 215 fps (Mikrotron, MC1311, Germany) to obtain the relative geometrical positions between the gecko's feet and the load-carrying sensor while the gecko was in locomotion. The positions of the feet were synchronized with the reaction forces by a LED light in the view frame of the camera. Around 2000 images were obtained for each trial and the locomotion behavior was observed using two points on the gecko's vertebrae (Figure 1(a)).

(v) Data processing. The gait information of a gecko moving on a vertical surface, obtained from a series of photos recorded by high speed camera, included the stride length $L_{\mathrm{s}}$, stride frequency $f_{\mathrm{s}}$, stance period $T_{\mathrm{st}}$, swing period $T_{\text {sw }}$, duty factor $D_{\mathrm{f}}$, attachment time $T_{\text {at }}$, detachment time $T_{\text {de }}$, etc. The valid contact of a gecko foot on the sensor, that is all parts of a foot or two feet in contact with one sensor were selected from the high speed recording, from which the maximum lateral reaction force $F_{\mathrm{L}}$, fore-aft force $F_{\mathrm{F}}$ and normal force $F_{\mathrm{N}}$ were extracted.

(vi) Statistics. An animal's reaction force might be influenced by many factors, such as body weight, stress and coping mode, locomotion behavior and environmental conditions, so the statistical analysis must be introduced to reveal the role of locomotion mechanics. We compared the difference in the data between groups by T-tests and set critical $P=0.05$ (SPSS Inc., Chicago, USA). All of the tested data were presented as means \pm standard deviations (mean \pm s.d.).

\section{Results}

When geckos moved on a vertical surface in upward, downward and leftward directions, the gait information and reaction forces generated by the left and right feet were compared using the T-test. The test results showed that there were no significant differences between the gecko moving upwards and downwards $(P>0.05)$, which may result from the symmetrical body structure of the gecko. However, there were obvious differences moving leftwards $(P<0.05)$. Thus, the opposite data generated by the left and right feet were combined when analyzing the gecko's upward and downward movement.

\subsection{Locomotion behaviors and gait}

Geckos moved vertically using a diagonal gait with velocity. Contralateral limbs moved nearly synchronously, while ipsilateral limbs moved in antiphase. Geckos extended or crimped their toes (when peeling from the terminal end) to attach to or detach from the substrate when they made the transformation from a stance period to a swing period.

(i) Moving upwards. Geckos moved upwards on a vertical surface at a speed of 0.09 to $0.72 \mathrm{~m} / \mathrm{s}$, with a stride length of 84.6 to $144.9 \mathrm{~mm}$, and a stride frequency of 0.73 $\mathrm{Hz}$ to $6.00 \mathrm{~Hz}$. The stride length and stride frequency were both significantly affected by speed (ANOVA, stride length: $R^{2}=0.364, \quad F=5.73, d f=1,10, P=0.038$; stride frequency: $\left.R^{2}=0.932, F=137.06, d f=1,10, P<0.001\right)$. Compared with the effects of stride length, the geckos increased their velocity largely by increasing their stride frequency (stride frequency $=6.783 \times v+0.489$ ) (Figure 2(a)). The duty factor of each foot was affected by speed (ANOVA, fore-feet: $R^{2}=0.432, F=16.71, d f=1,22, P=0.001$; hind-feet: $R^{2}=0.173$, $F=4.59, d f=1,22, P=0.043)$ and decreased with increasing velocity (Figure 2(b)). The times needed for attaching and detaching of fore- and hind-feet were not significantly affected by speed (ANOVA, $P>0.05$ ), the attachment and detachment time of the fore-feet were both shorter than for the hind-feet (Figure 3(a), Table 1).

(ii) Moving downwards. Geckos moved downwards on a vertical surface at a speed of $0.17 \mathrm{~m} / \mathrm{s}$ to $1.08 \mathrm{~m} / \mathrm{s}$, with a stride length of $71.9 \mathrm{~mm}$ to $136.6 \mathrm{~mm}$, and a stride frequency of $2.29 \mathrm{~Hz}$ to $7.68 \mathrm{~Hz}$. The stride length and stride frequency were both significantly affected by speed (ANOVA, stride length: $R^{2}=0.587, \quad F=11.38, \quad d f=1,8$, $P=0.010 ;$ stride frequency: $R^{2}=0.856, \quad F=47.67, d f=1,8$, $P<0.001)$. Compared with the effect of stride length, geckos increased their velocity largely by increasing their stride frequency (stride frequency=6.023 $\times v+1.679$ ) (Figure 2(c)). The duty factors of each foot were affected by speed (ANOVA, fore-feet: $R^{2}=0.540, F=21.13, d f=1,18, P=0.001$; hind-feet: $\left.R^{2}=0.416, F=12.82, d f=1,18, P=0.002\right)$ and decreased with increasing velocity (Figure $2(\mathrm{~d})$ ). The detachment times of each foot were not significantly affected by speed (ANOVA, $P>0.1$ ), the attachment and detachment time of the fore-feet were both longer than those of the hind-feet (Figure 3(b), Table 1).

(iii) Moving leftwards. Geckos moved leftwards on a vertical surface at a speed of 0.19 to $0.84 \mathrm{~m} / \mathrm{s}$, with a stride length of 85.1 to $141.0 \mathrm{~mm}$, and a stride frequency of $2.44 \mathrm{~Hz}$ to 6.94 $\mathrm{Hz}$. The speed does not relate to the stride length (ANOVA, $R^{2}=0.230, F=3.58, d . f .=1,12, P=0.083$ ), but relates to the stride frequency (ANOVA, $R^{2}=0.888, F=94.76, d f=1,12, P<0.001$ ). Thus the geckos increased their velocity by increasing their stride frequency (stride frequency $=7.238 \times v+0.737$ ) (Figure 2(e)). The duty factors of LF and RH were significantly affected by speed (ANOVA, LF: $R^{2}=0.291, F=4.92, d f=1,12$, $P=0.047$; RH: $\left.R^{2}=0.662, F=23.47, d f=1.12, P=0.001\right)$ and decreased with increasing velocity (LF: duty factor= $-0.313 \times v+0.823$; RH: duty factor $=-0.379 \times v+0.872)$. The duty factors of RF and LH were not significantly affected by speed (Figure 2(f)), nor were the attachment and detachment times of each foot (ANOVA, $P>0.463$ ), but the detachment times of fore- and hind-feet differed from one another and the 

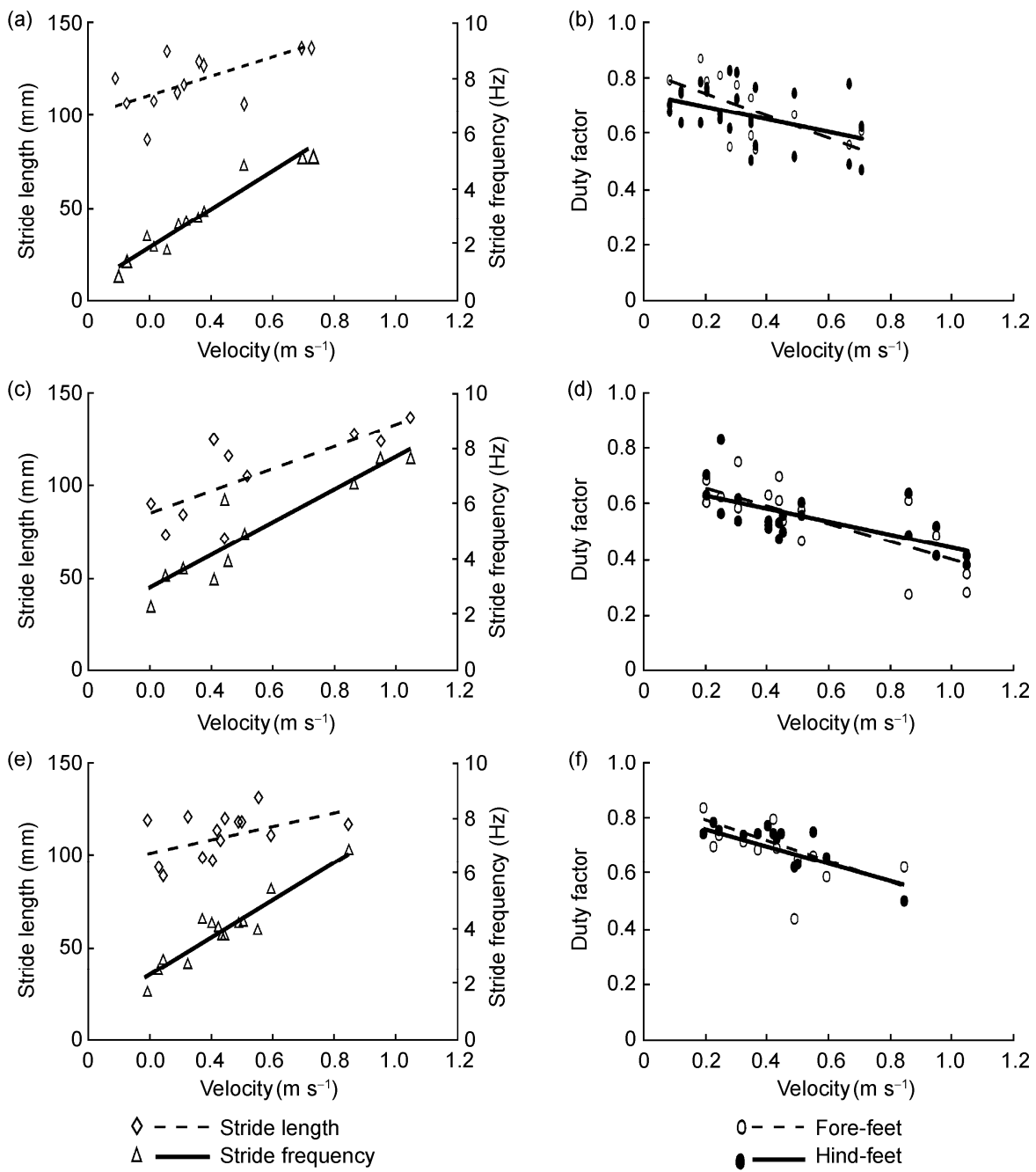

Figure 2 Stride lengths, stride frequency ((a), (c), (e)) and duty factors ((b), (d), (f)) vs. velocity for a G. Gecko moving in different directions on a vertical surface. (a) Stride length and stride frequency vs. velocity for a G. Gecko moving upwards on a vertical surface; (b) duty factors vs. velocity for a G. Gecko moving upwards on a vertical surface; (c) stride length and stride frequency vs. velocity for a G. Gecko moving downwards on a vertical surface; (d) duty factors vs. velocity for a G. Gecko moving downwards on a vertical surface; (e) stride length and stride frequency vs. velocity for a G. Gecko moving leftwards on a vertical surface; (f) duty factors vs. velocity for a G. Gecko moving leftwards on a vertical surface.

Table 1 Mean attachment and detachment times in a G. Gecko moving in different directions on a vertical surface

\begin{tabular}{|c|c|c|c|c|c|c|c|}
\hline & Foot & $T_{\mathrm{at}}(\mathrm{ms})$ & $T_{\mathrm{de}}(\mathrm{ms})$ & $T_{\mathrm{at}} / T_{\mathrm{st}}(\%)$ & $T_{\mathrm{at}} / T(\%)$ & $T_{\mathrm{de}} / T_{\mathrm{st}}(\%)$ & $T_{\mathrm{de}} / T(\%)$ \\
\hline \multirow{2}{*}{ Moving upward } & LF RF & $25.78 \pm 19.29$ & $102.13 \pm 78.54$ & $9.6 \pm 6.1$ & $6.1 \pm 3.3$ & $34.5 \pm 16.1$ & $22.5 \pm 10.7$ \\
\hline & LH RH & $33.72 \pm 21.63$ & $157.95 \pm 113.16$ & $12.2 \pm 7.5$ & $7.9 \pm 4.4$ & $43.1 \pm 27.3$ & $35.2 \pm 19.2$ \\
\hline \multirow{2}{*}{ Moving downward } & LF RF & $29.07 \pm 11.73$ & $70.47 \pm 59.74$ & $25.3 \pm 13.0$ & $13.4 \pm 5.6$ & $48.6 \pm 25.2$ & $27.2 \pm 14.0$ \\
\hline & LH RH & $20.23 \pm 9.65$ & $41.86 \pm 22.69$ & $17.1 \pm 9.7$ & $9.3 \pm 4.7$ & $36.1 \pm 21.5$ & $19.0 \pm 9.1$ \\
\hline \multirow{4}{*}{ Moving leftward } & LF & $24.25 \pm 7.33$ & $66.44 \pm 41.22$ & $12.7 \pm 3.6$ & $9.6 \pm 6.1$ & $36.1 \pm 21.7$ & $24.4 \pm 13.8$ \\
\hline & $\mathrm{RF}$ & $25.91 \pm 11.50$ & $59.81 \pm 33.18$ & $15.9 \pm 8.5$ & $9.9 \pm 5.7$ & $34.7 \pm 14.7$ & $21.5 \pm 10.6$ \\
\hline & $\mathrm{LH}$ & $29.57 \pm 9.05$ & $90.36 \pm 40.72$ & $16.8 \pm 5.6$ & $10.7 \pm 2.6$ & $49.6 \pm 21.0$ & $32.2 \pm 13.9$ \\
\hline & RH & $28.27 \pm 5.61$ & $78.41 \pm 52.90$ & $9.0 \pm 4.0$ & $6.8 \pm 2.6$ & $42.2 \pm 33.5$ & $30.1 \pm 23.2$ \\
\hline
\end{tabular}

attachment times of the fore-feet were slightly shorter than for the hind-feet (Figure 3(c), Table 1).

\subsection{Reaction force generated by each foot}

(i) Moving upwards. In the lateral direction, each foot pulled towards the midline of the body so that the left feet generated a lateral force to the right while the right feet generated a lateral force to the left. Lateral forces were 1.5 times the normal force. The feet must generate enough positive fore-aft forces to balance gravity and provide a driving force to move upward, thus the direction of the fore-aft 
(a)

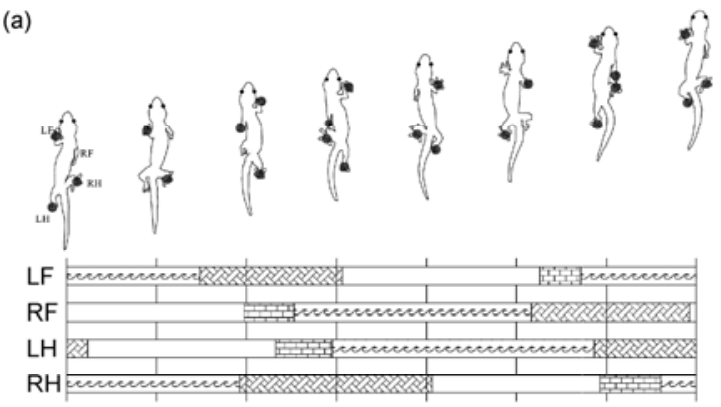

(c)
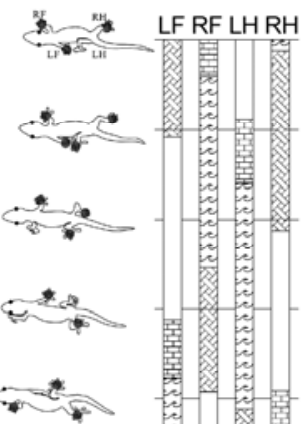

要
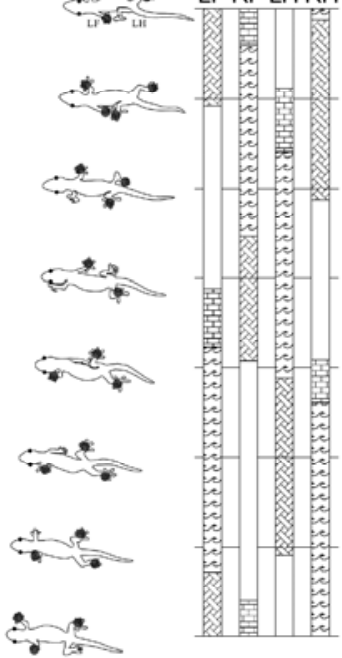

$\checkmark$ Swing period

宦琴琹Attachment

- Contact foot

Figure 3 Gait pattern vs. time during one stride of a G. Gecko moving in different directions on a vertical surface. (a) Moving upwards; (b) moving downwards; (c) moving leftwards.

force generated by each foot and the direction of locomotion were the same, which drove the gecko in an upward movement. The fore-aft forces of the fore- and hind-feet occupied $72.8 \%$ and $71.0 \%$ of the body weight, while the sum of the fore-aft forces generated by the two diagonal feet were about 1.45 times the body weight and 3 times the normal force. The negative normal forces (adhesive forces) generated by the fore-feet always pull the body towards the locomotion surface whereas the hind-feet pull the body towards the locomotion surface in the first half of the stance period and push the body away from the locomotion surface in the second half of the stance period (Figure 4(a)).

(ii) Moving downwards. The lateral forces generated by the fore-feet were less than that the forces generated by the hind-feet, and both deviated from the midline of the body. The direction of locomotion was in the direction of gravity, while the fore-aft forces generated by the fore- and hind-feet were opposite to gravity, and they took up $34.0 \%$ and $95.2 \%$ of the body weight. The sum of the fore-aft forces generated by the two diagonal feet was about 1.29 times the body weight and 5 times the normal force. The adhesive forces generated by the hind-feet always pull the body towards the locomotion surface, whereas the normal forces generated by the fore-feet pull the body towards the locomotion surface in the first half of the stance period and push the body away from the locomotion surface in the second half of the stance period (Figure 4(b)).

(iii) Moving leftwards. When the geckos moved leftwards on a vertical surface, the lateral forces generated by each foot both were opposite to gravity, the lateral forces of the right feet above the center-of-mass (COM) (RF and RH: occupying $108.7 \%$ and $122.0 \%$ of the body weight respectively) were larger than for the left feet below the COM (LF and LH: occupying $54.1 \%$ and $42.2 \%$ of the body weight), thus the weight was balanced primarily by the right feet above the COM. The sum of the fore-aft forces generated by the two diagonal feet was about 1.7 times the body weight and 5 times the normal force. The fore-aft force drived the gecko with a forward movement. The direction of the fore-aft forces generated by the fore-feet changed continuously in a stance period, in which initially the LF above the COM was opposite to the direction of locomotion and was then consistent with that, but the change of force generated by the RF was in the opposite direction to that generated by the LF. The fore-aft forces of the hind-feet were always unidirectional in the stance period, in which the $\mathrm{RH}$ generated a decelerating force and the RF generated an accelerating force in the fore-aft direction. The adhesive forces (negative normal forces) generated by the right feet above the COM pulled the body towards the locomotion surface, while the positive normal forces generated by the left feet below the COM pushed the body away from the locomotion surface (Figure 4(c)).

\subsection{Overturning impulse moments}

Static analyses of animals show that they need to overcome the unstable moment (overturning moment) induced by the deviation of the COM. This overturning moment is in proportion to the animal's weight and the deflected distance of the COM. Animals can use different methods (tail, foot and long toes, etc.) to balance this unstable moment. For example, 

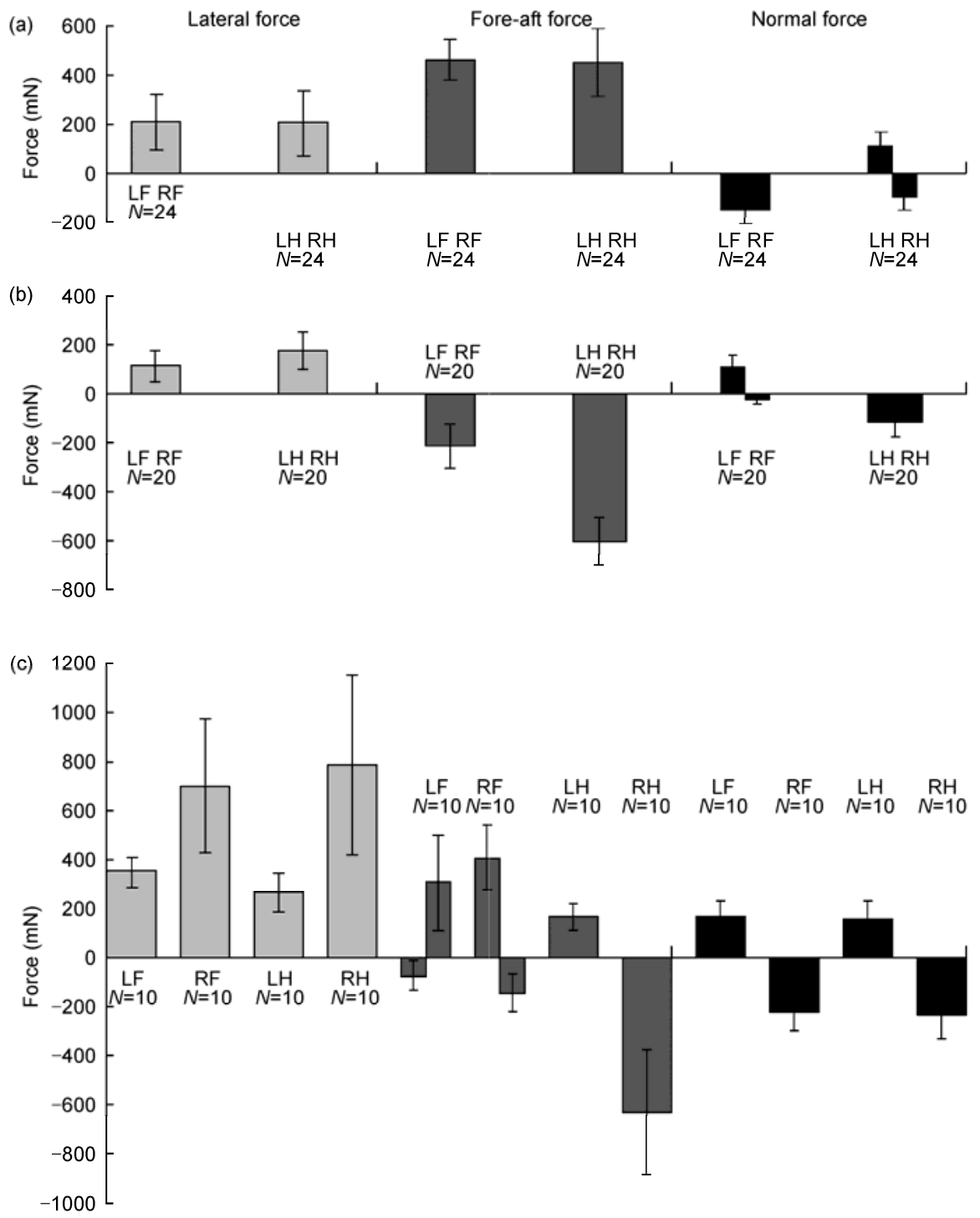

Figure 4 Mean maximum reaction forces of a single foot in a G. Gecko moving in different directions on a vertical surface. (a) Moving upwards; (b) moving downwards; (c) moving leftwards.

tree creepers use a tail to balance the overturning moment from below the COM [35]. When moving on floors and ceilings, geckos generated equal normal forces with the fore- and hind-feet in the same direction to support the body and balance the unstable moments $[16,18]$.

When geckos change direction on a vertical surface there is a distance between the COM and substrate. Thus, the overturning moment which can separate geckos from the vertical surface always exists (Figure 5(a), (b)). The results show that the feet above the COM always generate adhesive forces to pull the body toward the substrate to decrease the distance between the COM and the vertical surface. In moving upwards, the gravity impulse $I_{\mathrm{G}}$ was about 12 times the normal force impulse $I_{\mathrm{N}}$ generated by the feet above the COM. The ratio of the overturning arm of force $r$ to the stabilizing arm of force $R$ is $1 / 10$. The stabilizing impulse moment $M_{\mathrm{S}}$ generated by the normal force of the feet above the COM was less than the overturning impulse moment $M_{\mathrm{o}}$ induced by gravity. Downward and upward movement has similar conditions. The gravity impulse $I_{\mathrm{G}}$ was about 11 times the normal force impulse $I_{\mathrm{N}}$. The stabilizing impulse moment $M_{\mathrm{S}}$ was slightly smaller than the overturning impulse moment $M_{\mathrm{o}}$. For leftward movement, the stabilizing arm of force $R$ in leftward locomotion is less than that of upward and downward locomotion. The gravity impulse $I_{\mathrm{G}}$ was about 8 times the normal force impulse $I_{\mathrm{N}}$ generated by the feet above the COM. The stabilizing impulse moment $M_{\mathrm{S}}$ was larger than the overturning impulse moment $M_{\mathrm{O}}$ (Table 2).

\section{Discussion}

The reaction force and locomotion behaviors were meas- 
(a)

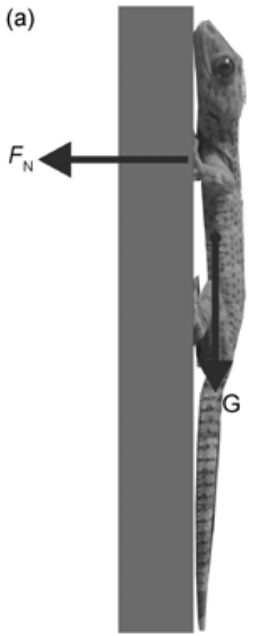

(b)

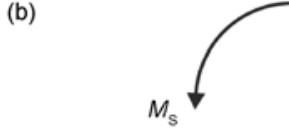

$M_{\mathrm{S}}=\int_{0}^{\Delta t} F_{\mathrm{N}} R \mathrm{dt}=R I_{\mathrm{N}}$ $I_{\mathrm{N}}=\int_{0}^{\Delta t} F_{\mathrm{N}} \mathrm{dt}$

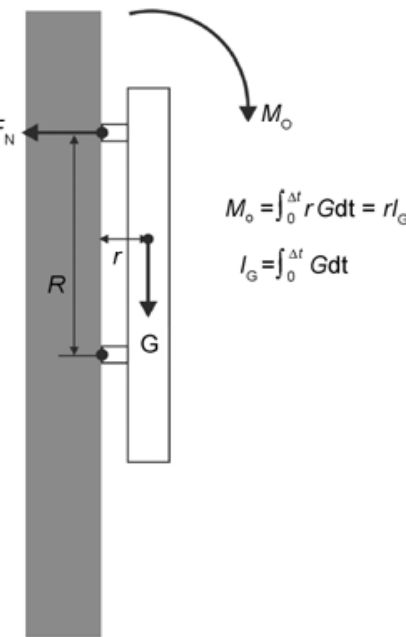

Figure 5 (a) Lateral view of normal reaction force during moving vertically; (b) lateral view of overturning $\left(M_{\mathrm{o}}\right)$ and stabilizing $\left(M_{\mathrm{S}}\right)$ impulse moments during moving in a different direction on vertical surface where $F_{\mathrm{N}}$ is the normal force generated by feet above the COM, $R$ is the arm of force of stabilizing, the integral of $F_{\mathrm{N}}$ and $G$ from 0 to $t$ represents the impulse $I_{\mathrm{N}}$ and $I_{\mathrm{G}}, G$ is the qravity, and $r$ is the distance of the COM to the vertical surface [15].

Table 2 Relationship between overturning impulse moment and stabilizing impulse moment in a G. Gecko moving in different directions on a vertical surface

\begin{tabular}{ccccc}
\hline Moving direction & $N$ & $I_{\mathrm{G}} / I_{\mathrm{N}}$ & $R / r$ & Impulse moment \\
\hline Upward & 20 & 12.45 & 10.02 & $M_{\mathrm{S}}<M_{\mathrm{o}}$ \\
Downward & 21 & 10.88 & 10.33 & $M_{\mathrm{S}}<M_{\mathrm{o}}$ \\
Leftward & 18 & 7.66 & 8.33 & $M_{\mathrm{S}}>M_{\mathrm{o}}$ \\
\hline
\end{tabular}

ured, when the G. Gecko moved on a vertical surface in upward, downward and leftward directions. The main difference between the three movements is the relationship between gravity and locomotion directions, which is, the reverse, uniform, and vertical. The results show that locomotion behavior and the reaction force of the G. Gecko change corresponding with different locomotion directions, and the locomotion behavior and reaction forces of different specific geckos were similar under the same form of motion.

\subsection{Locomotion behavior analysis}

The G. Gecko using a diagonal gait moved on a vertical surface in upward, downward and leftward directions, and the speed related to the gait information of the fore- and hind- feet, including stride length, stride frequency, duty factor and stance time, etc. When the gecko moved upwards, grav- ity always generated a deceleration force and the gecko needed to do work overcoming gravity, thus the velocity at $0.33 \pm 0.20 \mathrm{~m} / \mathrm{s}$ was the slowest for the three locomotion directions. In contrast, gravity always generated an acceleration force when the gecko moved downwards; therefore, the velocity was faster at $0.55 \pm 0.29 \mathrm{~m} / \mathrm{s}$. For leftward movement, there was no obvious transformation between potential energy and kinetic energy, thus the velocity lay between the other two locomotion directions at $0.43 \pm 0.17 \mathrm{~m} / \mathrm{s}$. The velocity of animals is influenced by stride length and stride frequency. The two species of geckos Gekko gecko (G. Gecko) and Hemidactylus garnotii (H. Gecko), tend to increase velocity by increasing stride frequency, even when they carry extra load, but other species of gecko (such as the Eublepharis macularius) tend to increase velocity by increasing stride length $[13,14]$. The test results show that when a gecko moved on a vertical surface in up- ward, downward and leftward directions, the stride length and stride frequency were both affected by the velocity. The gecko increased its velocity mainly by linear improvement in its stride frequency (Figure 2(a),(c),(e)), which is consistent with the test results of Zaaf and other authors. When geckos moved upwards and downwards, there were no significant differences in the duty factors of the left and right feet, and the duty factors of the fore- and hind-feet were equivalent. For leftward movement, the duty factor decreased with increasing velocity (Figure 2(b),(d),(f)). The attachment and detachment times both had obvious differences in the three directions and neither was significantly affected by the velocity (ANOVA, $P>0.05$ ), each occupying a certain proportion of the stride period. More specifically, geckos need a limited time to extend or crimp their toes, which may be due to achieving the least time with safe redundancy in the process of attachment and detachment. Thus, the gecko barely uses the decrease of attachment and detachment times to increase velocity (Table 1).

\subsection{Balancing overturning moment}

When geckos change directions on a vertical surface, the overturning moment caused by gravity can be reduced by decreasing the distance between the COM and the vertical surface, or can be balanced by the stabilizing moment generated by the adhesion force of the feet above the COM. The feet above the COM always generated negative normal force (adhesion force) to pull the body toward the vertical surface. 
When the geckos moved upwards and downwards, the feet below the COM generated positive normal force occasionally, but the value of this force was always less than the value of the negative normal force generated by the feet above the COM. Therefore, the net effect was to pull the body towards the locomotion surface to reduce the distance between the COM and substrate. A gecko which moves vertically uses a sprawled-posture, and needs to keep a minimum distance between its COM and the surface to maintain the sprawled-posture. There were no obvious differences in the arm of force of overturning moment $r$ for the three directions of movement. Thus the variation of the arm of force $r$ is limited, namely decrease of arms of force of the overturning moment $r$ has limited influence on reducing the overturning moment, so the gecko's only option is to keep the COM as close to the substrate as possible.

Adhesion forces generated by the feet play a key role in preventing turning and keeping still. However, a very large adhesion force will make it hard for geckos to detach themselves from the substrate, and will also make a greater impact on their locomotion. Geckos utilize the relationship between the arm of force of stabilizing moment $R$ and the arm of force of overturning moment $r$ ingeniously (Table 2). The overturning moment caused by gravity was balanced by small normal adhesion forces of the feet above the COM. Static analyses show that the stabilizing impulse moment caused by the feet above the COM must equal the overturning impulse moment caused by gravity. However, the experimental data show that there was some difference between the stabilizing impulse moment and overturning impulse moment (Table 2). It is supposed that, in reality, because a gecko is in a dynamic locomotion, there are a lot of influencing factors. For example, the inertia force caused by the motion, the swing of the tail in locomotion, and so on. Geckos can overcome the overturning moment and move steadily by using the feet or tail. It is not known whether the tail influences locomotion by its direct contact with the substrate or by its inertia [36]. This paper focuses on the function of the feet for stabilizing overturning moment.

\subsection{Differential foot function}

The reaction forces generated by the feet above the COM which is the fore-foot moving up, the hind-foot moving down and the right lateral foot moving to the left, are similar, the main differences are the force values. When the gecko moved upward, gravity was in the opposite direction to motion direction and a decelerating force is generated by gravity, so the positive driving forces generated by the feet had a similar direction to the motion direction to overcome gravity and drive the gecko upward. The fore- and hind-feet made the same contribution to balance gravity and drive motion (taking up $72.8 \%$ and $71.0 \%$ of the body weight respectively) (Figure 6(a)). In contrast, when the gecko moved downwards, the negative driving force generated by each foot is in the opposite direction to the direction of locomotion, and the deceleration force generated by the hind-foot above the COM took up $95.2 \%$ of the body weight and ensured stable and safe movement (Figure 6(b)). For leftward movement, gravity is perpendicular to the direction of locomotion, and the lateral forces of each foot are in the opposite direction to gravity. The lateral forces of the feet above the COM accounted for $108.7 \%$ and $122.0 \%$ of the body weight respectively and made the main contribution to balancing gravity. The fore-foot above the COM generated a positive driving force to accelerate locomotion in the first half of the stance period, and generated a negative driving force to decelerate locomotion in the second half of the stance period. The condition of the fore-foot below the COM was in contrast to that of fore-foot above the COM. The hind-foot above the COM always generated a negative driving force and the hind-foot below the COM always generated a positive driving force in the stance period. The driving ways, which accord with the change of the stance period, not only keep movement safe, but also greatly improve the efficiency of motion (Figure 6(c)).

Geckos move vertically using a sprawled-posture, the fore- and hind-feet play an important role in the lateral stability and maintenance of the sprawled-posture. A gecko twists its body with an S-shape while in motion, thus the foot needs to generate a homologous lateral force to balance the lateral inertia caused by the twisting. It is the premise of adhesion between the foot and substrate that suggests the gecko's limbs must pull towards the midline of the body. Lateral force is an important factor in making reliable adhesion with the vertical surface $[15,22,24]$. When the gecko moved upwards and downwards, each foot pulled toward the midline of the body and generated the necessary adhesion force to balance disturbances, such as the normal inertia force, overturning moment, etc. For leftward movement, only the feet above the COM pulled toward the midline of the body and the necessary adhesion forces were only generated by the feet above the COM. Therefore, when geckos changed direction on a vertical surface, the feet above the COM generated adhesion forces (negative normal forces) to pull the body toward the vertical surface. The normal force generated by feet below the COM change constantly to engage the requirements and balance the overturning moment in locomotion. The arms of force of overturning moment $r$ had no obvious differences in different directions, and the arms of force of stabilizing moment $R$ in leftward movement was less than that when moving upwards and downwards. Thereby the normal force of the feet above the COM of the gecko moving leftward was obviously larger than that of the gecko moving upwards and downwards (Figure 4 and Figure 6(a), (b), (c)).

Therefore, when the gecko moved upwards, downwards and leftwards on a vertical surface, the fore- and hind-feet were not significantly different in that they could achieve the same function. For example, the overturning moment 
(a)

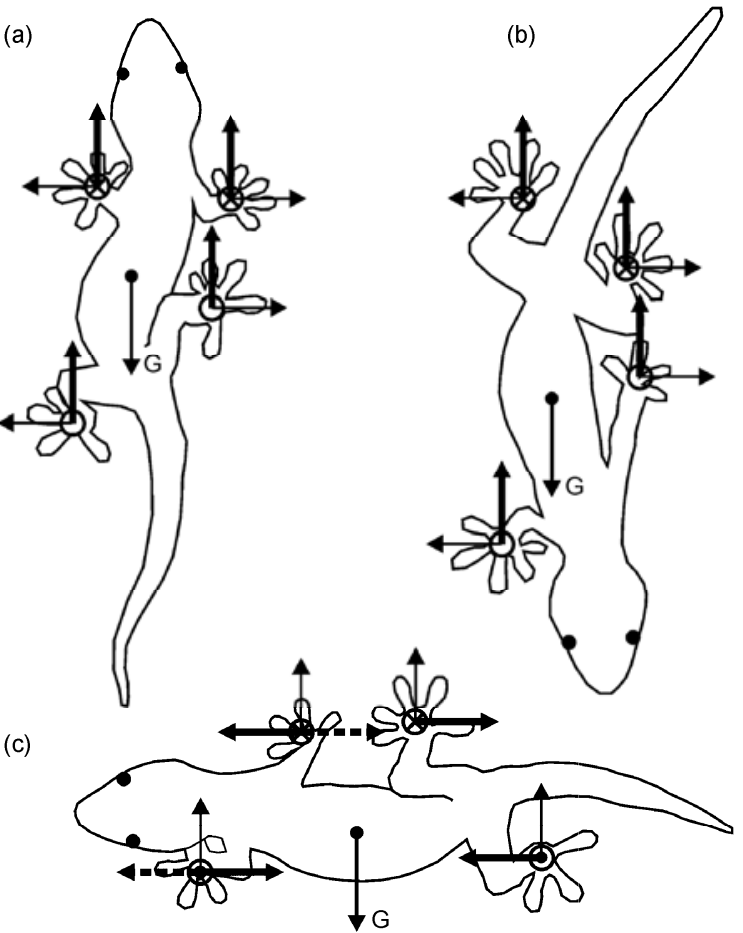

(d)
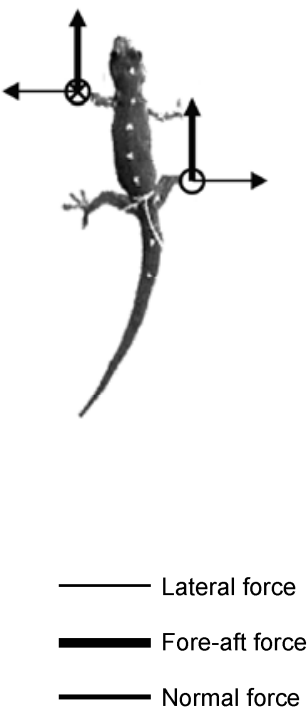

Figure 6 Direction of reaction forces of each foot in a G. Gecko moving in different directions on a vertical surface (a)-(c) and a $H$. Gecko running on level ground (d). (a) Single foot reaction force during a G. Gecko moving upwards. Circle with a $\times$ and dot in the center represents a vector that points away from and towards the reader; (b) single foot reaction force during a G. Gecko moving downwards; (c) single foot reaction force during a G. Gecko moving leftwards; (d) single foot reaction force during a H. Gecko's level running [15].

was mainly balanced by the fore-feet moving upwards, the hind-feet moving downwards and the right feet moving leftwards. However, when the gecko moved on a ceiling surface [18], the fore- and hind-feet have a significantly different function, the fore-feet play the main role of driving forward, and the hind-feet play the main role of keeping the motion steady. Following the synthetic analysis of the gecko moving on different surfaces or in different directions on one surface, it was found that there was no great difference in the function of the fore- and hind-feet, such as supporting the body, driving locomotion, balancing overturning, etc. Assuming that locomotion is required, geckos do not sedulously limit the function of each foot, or transform constantly and coordinate mutually the function of each foot to better complete locomotion in different direction on the same surface. On the other hand, geckos do properly limit the function of a foot on a different surface, such as on a ceiling surface where locomotion is only derived by the fore-feet. Although this may weaken the function of the foot, to a great extent, the adaptability of the gecko is improved in its natural environment and its survival is ensured. Meanwhile there is a good motor pattern in which geckos continually adjust the function of each foot for the change in locomotion environment. This can only inspire the structure design and control development for a gecko-like robot's legs and feet. It suggests that gecko-like robot control, and adjusting the function of each foot continually to adapt for different locomotion environments, will enable safe and efficient completion of specified tasks. For example the search and rescue tasks in extreme environments after a disaster and so on.

\subsection{Comparing the analyses for the Gekko Gecko (G. Gecko) and Hemidactylus garnotii (H. Gecko)}

The H. Gecko was a species of smaller gecko (weight: $1.9 \pm 0.7 \mathrm{~g}$, mean \pm s.d., $46-58 \mathrm{~mm}$ snout-vent length, $N=9$ ), its locomotion behaviors and dynamic movement upwards on a vertical surface were measured and analyzed by $\mathrm{Au}-$ tumn [15]. Comparing these analyses, there were differences and similarities between the H. Gecko and G. Gecko.

(i) Kinematics. The H. Gecko was smaller than the $G$. Gecko, its weight and snout-vent length occupied 1/32 and $1 / 3$ of the G. Gecko's, but their range of velocity had no obvious differences in the upward direction on a vertical surface. The stride length and stride frequency of the $G$. Gecko were significantly affected by velocity and the velocity increase was mainly a function of increasing stride frequency. The H. Gecko increased its velocity largely by increasing its stride length and the stride frequency did not relate to the velocity. The stance time, swing time and duty factor of the G. Gecko were significantly affected by velocity and increased with decreasing velocity. However, those of the H. Gecko were not significantly affected by velocity. 
This difference may be due to the G. Gecko being larger than the H. Gecko, thus it must have subtle control over the locomotive process to achieve safe and efficient locomotion.

The G. Gecko and H. Gecko moved vertically by using a diagonal gait at all speeds. The attachment time of the $G$. Gecko was larger than that of the H. Gecko, but the ratio of attachment time to stride time were virtually the same for the two species of geckos, and both the attachment and detachment times were not significantly affected by speed. Though there are obvious differences between different species of geckos in morphology, the characteristic of gait has some similarities which concurs with the results measured by Van Damme and Vanhooydonck et al. [5,37].

(ii) Single foot reaction force. The direction of fore-aft forces of both the G. Gecko and H. Gecko were opposite to the direction of gravity. The sum of the fore-aft forces generated by the two diagonal feet were about 1.45 and 1.92 times the body weight for the H. Gecko and G. Gecko, which showed that variation in the range of acceleration of the H. Gecko was greater than that of the G. Gecko, thus the acceleration ability of the H. Gecko may be better than the G. Gecko. The G. Gecko, which is larger than the H. Gecko, needs to decrease its lateral swing to reduce the lateral inertial impact and increase locomotive safety on a vertical surface. This may be because the ratio of the lateral force to body weight of the G. Gecko (0.33) is less than that of the H. Gecko (1.2) in the lateral direction. The adhesive forces (negative normal forces) of the fore-feet of the G. Gecko and H. Gecko took up $23 \%$ and $26 \%$ of their body weights. The hind-feet of the G. Gecko generated a negative normal force which took up $14 \%$ of the body weight in the first half of the stance period and a positive normal force which took up $18 \%$ of the body weight in the second half of the stance period. However, the hind-feet of the $H$. Gecko always generated a positive normal force which took up $26 \%$ of the body weight in the whole of the stance period. This change of hind-feet of the G. Gecko may be used to balance inertial perturbation which resulted from a bigger body in locomotion. Therefore, the first question for the $G$. Gecko is how to ensure its bigger body moves safely on a vertical surface (Figure 6(a), (d) and Figure 7).

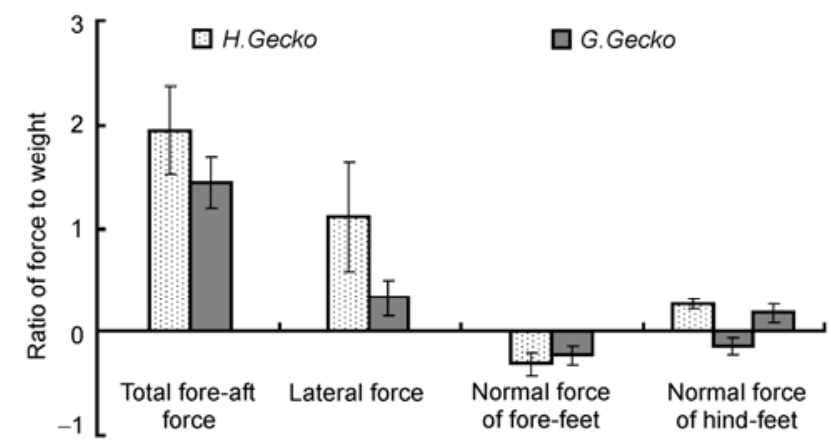

Figure 7 Comparison diagrams of ratio of reaction forces to weight for a G. Gecko and a H. Gecko moving on a vertical surface.
This work was supported by the Funding for Outstanding Doctoral Dissertation in Nanjing University of Aeronautics and Astronautics (BCXJ10-10), the National Hi-Tech Research \& Development Program of China (2007AA04Z201) and the National Natural Science Foundation of China (60910007, 50705043, 50975140 and 30700068).

1 Dickinson M H, Farley C T, Full R J, et al. How animals move: An integrative view. Science, 2000, 288: 100-106

2 Moermond T C. Habitat constraints on the behavior, morphology, and community structure of anolis lizards. Ecology, 1979, 60: 152-164

3 Losos J B. The evolution of form and function: Morphology and locomotor performance in West Indian Anolis lizards. Evolution, 1990, 44: 1189-1203

4 Sinervo B, Losos J B. Walking the tight rope: Arboreal sprint performance among Sceloporus occidentalis lizard population. Ecology, 1991, 72: 1225-1233

5 Van Damme R, Aerts P, Vanhooydonck B. No trade-off between sprinting and climbing in two populations of the Lizard Podarcis hispanica. Biol J Linn Soc, 1997, 60: 493-503

6 Zaaf A, Herrel A, Aerts P, et al. Morphology and morphometrics of the appendicular musculature in geckoes with different locomotor habits (Lepidosauria). Zoomorphology, 1999, 119: 9-22

7 Miles D B, Fitzgerald L A, Snell H L. Morphological correlates of locomotor performance in hatchling Amblyrhynchus cristatus. Oecologia, 1995, 103: 261-264

8 Rewcastle S C. Stance and gait in tetrapods: An evolutionary scenerio. Symp Zool Soc Lond, 1981, 48: 239-267

9 Bauer A M, Russell A P, Powell G L. The evolution of locomotor morphology in Rhoptropus (Squamata: Gekkonidae): Functional and phylogenetic considerations. Afr J Herpetol, 1996, 45: 8-30

10 Cartmill M. Functional vertebrate morphology. Cambridge, MA: Harvard University Press, 1985

11 Peterson J A. The locomotion of Chamaeleo (Reptilia: Sauria) with particular reference to the forelimb. Symp Zool Soc Lond, 1984, 202: 1-42

12 Losos J B, Walton B M, Bennett A F. Trade-offs between sprinting and clinging ability in Kenyan Chameleons. Funct Ecol, 1993, 7: 281-286

13 Irschick D J, Vanhooydonck B, Herrel A, et al. Effects of loading and size on maximum power output and gait characteristics in geckos. J Exp Biol, 2003, 206: 3923-3934

14 Zaaf A, Van Damme R, Herrel A, et al. Spatio-temporal gait characteristics of level and vertical locomotion in a ground-dwelling and a climbing gecko. J Exp Biol, 2001, 204: 1233-1246

15 Autumn K, Hsieh S T, Dudek D M, et al. Dynamics of geckos running vertically. J Exp Biol, 2006, 209: 260-272

16 Chen J J, Peattie A M, Autumn K, et al. Differential leg function in a sprawled-posture quadrupedal trotter. J Exp Biol, 2006, 209: 249-259

17 Li H K, Dai Z D, Shi A J, et al. Angular observation of joints of geckos moving on horizontal and vertical surfaces. Chinese Sci Bull, 2009, 54 : 592-598

18 Wang Z H, Wang J T, Ji A H, et al. Gecko's movement behavior and dynamics research on ceiling surface. Chinese Sci Bull, 2010, 55, 3356-3362

19 Autumn K, Peattie A. Mechanisms of adhesion in Geckos. Soc Integ Comp Biol, 2002, 42: 1081-1090

20 Arzt E, Gorb S, Spolenak R. From micro to nano contacts in biological attachment devices. Proc Natl Acad Sci USA, 2003, 100: 10603-10606

21 Chen B B, Wu P D, Gao H. Hierarchical modeling of attachment and detachment mechanisms of gecko toe adhesion. Proc R Soc A, 2008, 464: 1639-1652

22 Autumn K, Liang T A, Flsieh S T, et al. Adhesive force of a single gecko foot-flair. Nature, 2000, 405: 681-685

23 Pesika N S, Tian Y, Zhao B X, et al. Peel-zone model of tape peeling based on the gecko adhesive system. J Adhes, 2007, 83: 383-401

24 TianY, Pesika N, Zeng H B, et al. Adhesion and friction in gecko toe attachment. Proc Natl Acad Sci USA 2006, 103: 19320-19325

25 Jagota A, Bennison S J. Mechanics of adhesion through a fibrillar microstructure. Integr Comp Boil, 2002, 42: 1140-1145 
26 Huber G, Mantz H, Spolenak R, et al. Evidence for capillarity contributions to gecko adhesion from single spatula nanomechanical measurements. Proc Natl Acad Sci USA, 2005, 102: 16293 16296

27 Bhushan B, Sayer R A. Gecko feet: Natural attachment systems for smart adhesion. In: Bhushan B, Tomitori M, Fuchs H, eds. Applied Scanning Probe Methods VII. Heidelberg: Springer Berlin Heidelberg, 2007. 41-76

28 Irschick D J, Austin C C, Petren K, et al. A comparative analysis of clinging ability among pad-bearing lizards. Biol J Linn Soc, 1996, 59: 21-35

29 Santos D, Spenko M, Parness A, et al. Directional adhesion for climbing: Theoretical and practical considerations. J Adhesion Sci Tech, 2007, 21: 1317-1341

30 Gorb S, Varenberg M, Peressadko A, et al. Biomimetic mushroomshaped fibrillar adhesive microstructure. J R Soc Interface, 2007, 4: 271-275

31 Aksak B, Murphy M P, Sitti M. Adhesion of biologically inspired ver- tical and angled polymer microfiber arrays. Langmuir, 2007, 23: 33223332

32 Qu L T, Dai L M, Stone M, et al. Carbon nanotube arrays with strong shear binding-on and easy normal lifting-off. Science, 2008, 322: 238 $-242$

33 Van Damme R, Aerts P, Vanhooybonck B. Variation in morphology gait characteristics and speed of locomotion in two populations of lizards. Biol J Linn Soc, 1998, 63: 409-427

34 Zhang Z J, Ji A H, Wang Z Y, et al., Three-dimensional sensor for measuring gecko s ground reaction force. Chin J Sens Actu, 2007, 20: $1271-1274$

35 Norberg R A. Tree creeper climbing: Mechanics, energetics and structural adaptations. Ornis Scand. 1986, 17: 191-209

36 Walter R M, Carrier D R. Scaling of rotational inertia in murine rodents and two species of lizard. J Exp Biol, 2002, 205: 2135-2141

37 Vanhooydonck B, Van Damme R, Aerts P. Variation in speed, gait characteristics and microhabitat use in lacertid lizards. J Exp Biol, 2002, 205: 1037-1046

Open Access This article is distributed under the terms of the Creative Commons Attribution License which permits any use, distribution, and reproduction in any medium, provided the original author(s) and source are credited. 